\title{
Estudo Prospectivo das Repercussões de Baixas Doses de Remifentanil na Função Sinoatrial e na Condução e Refratariedade Cardíaca*
}

\author{
Prospective Study on the Repercussions of Low Doses of Remifentanil on Sinoatrial \\ Function and in Cardiac Conduction and Refractory Period
}

\author{
Simone Soares Leite, TSA ${ }^{1}$, Elizabeth Bessadas Penna Firme, TSA², Márcia Santana Bevilaqua, TSA ${ }^{3}$, \\ Leonel dos Santos Pereira, TSA ${ }^{4}$, Jacob Atié
}

\section{RESUMO}

Leite SS, Firme EBP; Bevilaqua MS, Pereira LS, Atié J - Estudo Prospectivo das Repercussões de Baixas Doses de Remifentanil na Função Sinoatrial e na Condução e Refratariedade Cardíaca. JUSTIFICATIVA E OBJETIVOS: O remifentanil é um opióide com início e término de ação rápidos, cujo uso em procedimentos de curta duração vem se propagando nos últimos anos. Entre os efeitos colaterais descritos, há relatos de bradicardia e assistolia. $O$ objetivo deste estudo foi avaliar os efeitos desse fármaco na condução e refratariedade cardíaca, em humanos.

MÉTODO: Estudo prospectivo de 16 pacientes, entre 18 e 65 anos, de ambos os sexos, ASA I a III, submetidos a estudo eletrofisiológico intracardíaco eletivo. Foram excluídos os pacientes com doença do nódulo sinoatrial e os portadores de bloqueios cardíacos graves. No laboratório de eletrofisiologia, os pacientes foram inicialmente sedados com midazolam $\left(0,03 \mathrm{mg} \cdot \mathrm{kg}^{-1}\right)$, após 5 minutos (MO) avaliou-se o grau de sedação de intensidade de dor, pressões arteriais sistólica e diastólica, freqüências cardíaca e respiratória e saturação de oxigênio. O eletrofisiologista avaliou as variáveis de condução cardíaca (duração do QRS, intervalos $A A, A H, H V$ e $P A)$, o tempo de recuperação do nódulo sinoatrial e as variáveis de refratariedade cardíaca (período refratário do átrio direito, período refratário do ventrículo direito e período refratário do nódulo atrioventricular). Após as medidas iniciais o remifentanil foi introduzido (bolus de 0,5 $\mu \mathrm{g} \cdot \mathrm{kg}^{-1}+$ infusão de $0,05 \mu \mathrm{g} \cdot \mathrm{kg}^{-1} \cdot \mathrm{min}^{-1}$ ) e após 20 minutos as mesmas variáveis foram reavaliadas (M1).

RESULTADOS: Observou-se diminuição das pressões sistólica e diastólica $(p=0,0001)$ entre Mo e M1, sem diferença estatística sig-

\footnotetext{
${ }^{*}$ Pesquisa realizada no Hospital Clementino Fraga Filho (HUCFF) da Faculdade de Medicina da Universidade Federal do Rio de Janeiro (FM/UFRJ), Rio de Janeiro, $R J$
}

1. Médica Assistente do Serviço de Anestesia do HUCFF/UFRJ; Co-Responsável pelo CET do HUCFF/UFRJ; Chefe do Serviço de Anestesia do Hospital Maternidade Carmela Dutra

2. Médica Assistente do Serviço de Anestesia do HUCFF/UFRJ; Co-Responsável pelo CET do Hospital dos Servidores do Estado do Rio de Janeiro

3. Médica Assistente do Serviço de Anestesia do HUCFF/UFRJ; Co-Responsável pelo CET do Hospital dos Servidores do Estado do Rio de Janeiro 4. Co-Responsável pelo CET do HUCFF/UFRJ; Doutor em Medicina do Departamento de Cirurgia (Setor Anestesiologia) da FM/UFRJ; Professor Adjunto do Departamento de Cirurgia (Especialidade Anestesia) da UFRJ; Chefe do Serviço de Anestesia do HUCFF/UFRJ

5. Chefe do Setor de Arritmias Cardíacas do HUCFF/UFRJ e da Clínica São Vicente da Gávea; Professor Adjunto do Departamento de Cardiologia da UFRJ;

Apresentado (Submitted) em 15 de maio de 2006

Aceito (Accepted) para publicação em 12 de junho de 2007

Endereço para correspondência (Correspondence to):

Simone Soares Leite

Rua Marques de Paraná, 128/706 - Flamengo

22230-030 Rio de Janeiro, RJ

E-mail: sslsimone@bol.com.br

() Sociedade Brasileira de Anestesiologia, 2007 nificativa da freqüência respiratória ou da saturação de oxigênio. Houve aumento do intervalo átrio-His $(p=0,006)$ e do tempo de recuperação do nódulo sinoatrial $(p=0,0004)$, do período refratário do átrio direito $(p=0,001)$ e do período refratário do nódulo atrioventricular $(p=0,0001)$, porém não houve diminuição da freqüência cardíaca basal entre MO e M1.

CONCLUSÕES: O remifentanil alterou as variáveis eletrofisiológicas cardíacas, o que em doses maiores que as estudadas poderiam causar bradicardia sinusal, assistolia e distúrbios de condução. Unitermos: ANALGÉSICOS, Opióide: remifentanil, CIRURGIA, Cardíaca: ablação; COMPLICAÇÕES: assistolia, bradicardia; FISIOLOGIA, Cardiovascular: condução, refratariedade.

\section{SUMMARY}

Leite SS, Firme EBP, Bevilaqua MS, Pereira LS, Atié J - Prospective Study on the Repercussions of Low Doses of Remifentanil on Sinoatrial Function and in Cardiac Conduction and Refractory Period. BACKGROUND AND OBJECTIVES: Remifentanil is an opiod with fast onset of action and short acting, and its use in short-duration procedures has increased in the last few years. Bradycardia and asystole are among the side effects reported. The objective of this study was to evaluate the effects of this drug in cardiac conduction and refractory period in human beings.

METHODS: A prospective study with 16 patients, ages 18 to 65, both genders, ASA I to III, undergoing elective intracardiac electrophysiological study, was undertaken. Patients with disorders of the sinoatrial node and those with severe cardiac blocks were excluded. In the laboratory of electrophysiology, patients were sedated with midazolam (0.03 mg. $\left.\mathrm{kg}^{-1}\right)$ after 5 minutes the degree of sedation and degree of pain, systolic and diastolic blood pressure, heart rate and respiratory rate, and oxygen saturation were evaluated. The electrophysiologist evaluated cardiac conduction (duration of the QRS complex, and $A A, A H, H V$, and PA intervals), duration of sinoatrial node recovery, and cardiac refractory period (refractory period of the right atrium, right ventricle, and atrioventricular node). After the initial measurements, remifentanil was administered (bolus of $0.5 \mu \mathrm{g} \cdot \mathrm{kg}^{-1}+$ infusion of $0.05 \mu \mathrm{g} \cdot \mathrm{kg}^{-1} \cdot \mathrm{min}^{-1}$ ) and, after 20 minutes, the same parameters were evaluated.

RESULTS: There was a reduction in systolic and diastolic blood pressure ( $p=0.0001)$ between MO and M1, and significant differences in respiratory rate and oxygen saturation, which were not statistically significant. The atrium-His interval $(p=0.006)$, recovery time of the sinoatrial node $(p=0.0004)$, refractory period of the right atrium $(p=0.001)$, and refractory period of the sinoatrial node ( $p=$ $0.0001)$ were all increased; however, there were no differences in heart rate between $\mathrm{MO}$ and $\mathrm{M} 1$.

CONCLUSIONS: Remifentanil changes cardiac electrophysiological parameters and, in doses higher than the ones used in this study, can cause sinus bradycardia, asystole, and conduction defects.

Key Words: ANALGESICS, Opioid: remifentanil; COMPLICATIONS, Heart Arrest; Arrhythmia: Bradycardia; PHYSIOLOGY, Cardiovascular system: Heart conduction system; SURGERY, Cardiac; Catheter ablation. 


\section{INTRODUÇÃO}

O remifentanil é um agonista seletivo do receptor mu, com potência semelhante ao fentanil. Sua estrutura química apresenta uma ligação metil-éster-propanóica que é hidrolisada pelas esterases plasmáticas inespecíficas e pelas teciduais (carboxiesterase), resultando em uma duração de ação curta e previsível, sem acúmulo de efeito com doses repetidas ou quando administrado em infusão venosa contínua (meia-vida contexto-dependente de três minutos). Também possui volumes de distribuição nos compartimentos centrais e totais muito baixos, apresentando meia-vida beta muito menor que os outros opióides, além de uma pequena meia-vida $\mathrm{K}_{\mathrm{e}} \mathrm{O}$, o que the confere um rápido início de ação ${ }^{1-9}$. Esse perfil farmacocinético e farmacodinâmico é muito atraente, sobretudo para a realização de procedimentos rápidos. Entretanto, o seu uso está associado à instabilidade hemodinâmica, com hipotensão arterial e bradicardia ${ }^{10-14}$. Além disso, há na literatura o relato de dois casos de assistolia após o uso de remifentanil na indução anestésica ${ }^{15,16}$.

Os relatos de bradicardia foram observados principalmente nos pacientes em uso de fármacos antagonistas do receptor beta adrenérgico, nos pacientes com bradicardia preexistente e naqueles que receberam dose em bolo do

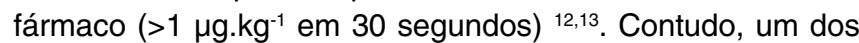
casos de assistolia descrito na literatura ocorreu em um paciente jovem, sem doenças associadas, em quem não foi administrada dose em bolus ${ }^{16}$.

O objetivo deste estudo foi avaliar, em humanos, se o remifentanil exerce efeito na condução e refratariedade cardíaca que justifique as bradicardia e assistolia observadas por outros autores.

\section{MÉTODO}

Após aprovação do Comitê de Ética em Pesquisas do Hospital Universitário Clementino Fraga Filho e obtenção de consentimento livre e informado, foram estudados de forma prospectiva, 16 pacientes adultos, de ambos os sexos, escalados para realização eletiva de estudo eletrofisiológico intracardíaco percutâneo, ablação de disritmias por cateter de radiofreqüência ou ambos.

A técnica anestésica empregada foi sedação e analgesia, e o componente sedativo e amnésico escolhido foi o midazolam. Os critérios de inclusão adotados foram idade entre 18 e 65 anos, estado físico ASA I a III e peso menor que $100 \%$ acima do ideal.

Os critérios de exclusão foram pacientes usuários habituais de benzodiazepínicos, antidepressivos, drogas ilícitas ou estimulantes do sistema nervoso central; os em uso habitual de antagonistas dos receptores beta ou fármacos agonistas do receptor alfa $_{2}$; pacientes que foram submetidos à anestesia ou usaram opióides nas últimas 48 horas; pacientes em uso de amiodarona (quando suspensa há menos de 45 dias); pacientes com Miastenia Gravis ou glaucoma de ângulo fechado e os considerados com "estômago cheio"; pacientes com distúrbios psiquiátricos, doença pulmonar grave, insuficiência cardíaca descompensada, angina instável ou infarto agudo do miocárdio nas últimas seis semanas; pacientes com bloqueios cardíacos prévios graves; pacientes com nefropatias ou hepatopatias.

Em todos os pacientes submetidos ao estudo os fármacos antidisrítmicos foram suspensos por no mínimo cinco meiasvidas (exceto a amiodarona).

O estudo eletrofisiológico intracardíaco foi realizado no laboratório de eletrofisiologia cardíaca do Hospital Clementino Fraga Filho.

Nenhum paciente recebeu medicação pré-anestésica.

Após monitorização com eletrocardiograma de 12 derivações com o sistema EPTRACER V0.73 (ECG), oxímetro de pulso digital, pressão arterial não-invasiva (PANI) e capnógrafo (aferidos a partir do monitor Cardiocap Ultima Datex Ohmeda), foi realizada venóclise no membro superior esquerdo com cateter $18 \mathrm{G}$ e administrados $20 \mathrm{~mL} \cdot \mathrm{kg}^{-1}$ de solução de Ringer com lactato, em 10 a 15 minutos. Subseqüentemente, a infusão foi diminuída a uma velocidade mínima para manter a veia patente. Ao equipo de infusão foram adicionados um conector em $Y$ (Polifix ${ }^{\circledR}$-B Braun) e uma torneira de três vias (TAP-3 Embramed) interposta entre o conector e o cateter venoso.

A seguir, foram administrados $0,03 \mathrm{mg} \cdot \mathrm{kg}^{-1}$ (peso ideal) de midazolam, antes das punções femorais realizadas pelo eletrofisiologista.

No M0 (5 minutos após a administração do midazolam) foi avaliado o grau de sedação pela escala de Ramsay, intensidade de dor por uma escala subjetiva de 0 a $4(0=$ nenhuma dor e 4 = maior dor que já sentiu na vida), pressões arteriais sistólica (PS) e diastólica (PD), freqüência cardíaca (FC) sem eletroestimulação, freqüência respiratória (FR), saturação arterial de oxigênio $\left(\mathrm{SpO}_{2}\right)$, pela oximetria de pulso, com oxigenoterapia suplementar (5 L.min ${ }^{-1}$ de $\mathrm{O}_{2}$ sob máscara de Hudson), $\mathrm{CO}_{2}$ expirado por capnografia $\left(\mathrm{P}_{\mathrm{ET}} \mathrm{CO}_{2}\right)$, as variáveis de condução cardíaca (duração do QRS, intervalos AA, AH, HV e PA), função do nódulo sinoatrial (tempo de recuperação do nódulo sinoatrial) e de refratariedade cardíaca (período refratário do átrio direito, do ventrículo direito e do nódulo atrioventricular). Ver glossário para definições.

O estudo eletrofisiológico basal foi realizado pelo registro simultâneo do eletrocardiograma de 12 derivações e dos sinais intracavitários (EPTRACER V0.73) que foram captados por três ou quatro cateteres-eletrodos transvenosos (Biosense Webster ${ }^{\circledR}$ quadripolar $6 \mathrm{~F}$ ) localizados na região alta de átrio direito, adjacente ao feixe de His, na ponta de VD e no seio coronário. A duração do $Q R S$ e os intervalos basais (intervalos AA, PR, PA, AH, HV e QT) foram aferidos durante o ritmo sinusal com velocidade de registro de $100 \mathrm{~mm} . \mathrm{s}^{-1}$. Os PREAD, PREVD e PRENAV foram aferidos por extraestímulos decrescentes inseridos sobre estimulação con- 


\section{ESTUDO PROSPECTIVO DAS REPERCUSSÕES DE BAIXAS DOSES DE REMIFENTANIL NA FUNÇÃO SINOATRIAL E NA CONDUÇÃO E REFRATARIEDADE CARDÍACA}

tínua nos ciclos de 600, 500 e 430 ms. O tempo de recuperação do nódulo sinoatrial (TRNS) foi determinado por estimulação atrial direita com comprimentos de ciclo de 600 , 550, 430 e 380 ms, por 1 minuto em cada nível e com velocidade de registro de $100 \mathrm{~mm} \cdot \mathrm{s}^{-1}$. Dez ciclos foram analisados após o término da estimulação, e o maior dentre esses intervalos pós-estimulação foi utilizado para calcular o TRNS. A configuração da onda P no ECG de superfície e os eletrogramas intra-atriais foram utilizados para verificar que o primeiro batimento após o término da estimulação foi realmente de origem sinusal.

Os pacientes que não apresentaram, em M0, os intervalos PA, AH e HV dentro da faixa de normalidade das medidas padronizadas no laboratório foram excluídos do estudo. Assim como os pacientes com síndrome de WPW cujo PRE anterógrado da via acessória foi menor que o do NAV.

Após as medidas iniciais, o remifentanil foi iniciado com bolus por via venosa de $0,5 \mu \mathrm{g} \cdot \mathrm{kg}^{-1}$ (peso ideal), em $30 \mathrm{se}$ gundos, seguido da infusão venosa contínua (na dose de $0,05 \mu \mathrm{g} \cdot \mathrm{kg}^{-1} \cdot \mathrm{min}^{-1}$ de peso ideal), de modo a manter um grau de sedação 2 a 4, pela escala de Ramsay e intensidade de 0 a 1 de dor. A dose do remifentanil podia ser ajustada em até mais ou menos $50 \%$ à inicial para atingir tais objetivos. Os pacientes que não atingiram esses objetivos receberam propofol, em infusão contínua, com ou sem bolus de $0,5 \mathrm{mg} \cdot \mathrm{kg}^{-1}$ (peso ideal) e foram afastados do estudo.

Após a avaliação dos parâmetros hemodinâmicos e respiratórios (PANI, $\mathrm{FC}, \mathrm{FR}, \mathrm{P}_{\mathrm{ET}} \mathrm{CO}_{2}$ e $\mathrm{SpO}_{2}$ ) a dose do remifentanil foi diminuída em até $50 \%$ da dose inicial, quando a pressão arterial sistólica foi menor que $15 \mathrm{mmHg}$ da inicial ou menor que $80 \mathrm{mmHg}$, ou quando a freqüência respiratória foi menor que $8 \mathrm{irpm}$ ou a $\mathrm{SpO}_{2}$ menor que 94\% (com oxigênio suplementar) ou nos casos em que freqüência cardíaca foi menor que $40 \mathrm{bpm}$.

As mesmas variáveis foram reavaliadas em M1 (após 20 minutos de infusão ajustada de remifentanil).

Após avaliação de todas as variáveis propostas no estudo, a anestesia foi mantida a critério do anestesiologista responsável pela mesma. Não foi estudada a indutibilidade da taquicardia sustentada.

A análise comparativa das variáveis em M0 e M1 foi realizada pelo teste $t$ de Student pareado e foram consideradas diferenças significativas quando $p<0,05$.

\section{RESULTADOS}

As características dos pacientes estudados encontram-se na tabela I.

A indicação do procedimento foi o diagnóstico eletrofisiológico e ablação por cateter de radiofreqüência de taquicardia reentrante nodal em sete casos e de síndrome de Wolff-Parkinson-White nos outros nove.

O tempo médio de duração do procedimento foi de $78 \pm 25$ minutos (máximo de 128 e mínimo de 40 minutos) e a dose média total de remifentanil por procedimento foi de $276 \pm$ $115 \mu \mathrm{g}$ (máximo de 520 e mínimo de $140 \mu \mathrm{g}$ ), com uma média de $3,45 \pm 0,12 \mu \mathrm{g} \cdot \mathrm{min}^{-1}$.

O grau de sedação alcançado foi satisfatório em todos os casos. Com grau 2 a 3 pela Escala de Ramsay no Mo e de 3 a 4, no M1 (Tabela II).

A intensidade de dor pela escala subjetiva usada foi de 0 a 2 em M0 e de 0 a 1 em M1 (Tabela III). Essas variáveis foram consideradas satisfatórias e não foi necessária a associação de propofol em nenhum dos casos.

Em relação às variáveis hemodinâmicas e respiratórias (PANI, FC, FR, $\mathrm{P}_{\mathrm{ET}} \mathrm{CO}_{2}$ e $\mathrm{SpO}_{2}$ ), avaliadas em $\mathrm{M0}$ e $\mathrm{M} 1$, foram observadas diferenças estatísticas significativas, apenas em relação às pressões arteriais sistólicas e diastólicas (Tabela IV). Quanto à freqüência cardíaca não foi constatada diferença significativa entre $\mathrm{MO}$ e $\mathrm{M} 1$.

Em relação às variáveis de condução cardíaca (duração do QRS, intervalos AA, AH, HV e PA), foi observado aumento da duração do QRS e do intervalo AH (Tabelas V e VI). Não houve variação estatística significativa entre $M 0$ e $M 1$, nas medidas dos intervalos AA, HV e PA.

Também foi observado aumento do TRNS (Tabela VII).

Tabela I - Características dos Pacientes $(n=16)$

\begin{tabular}{lc}
\hline Variáveis & \\
\hline Idade $(\text { anos })^{*}$ & $43 \pm-6(25-26)$ \\
Peso $(\mathrm{kg})^{\star}$ & $66 \pm-8(52-85)$ \\
Altura $(\mathrm{m})^{\star}$ & $1,63 \pm-0,10(1,51-1,83)$ \\
Sexo** & $10(62,5 \%)$ \\
Masculino & $6(38,5 \%)$ \\
Feminino & $10(62,5 \%)$ \\
Estado físico** & $6(38,5 \%)$ \\
ASA II & \\
ASA III &
\end{tabular}

* Valores expressos em Média \pm DP ou Número.

** Número Total (\%).

Tabela II - Escala de Ramsay, em M0 e M1

\begin{tabular}{lcc}
\hline Escala de Ramsay & $\mathrm{MO}^{*}$ & $\mathrm{M}^{*}$ \\
\hline 1 & 0 & 0 \\
2 & 6 & 0 \\
3 & 10 & 4 \\
4 & 0 & 12 \\
5 & 0 & 0 \\
6 & 0 & 0 \\
\hline
\end{tabular}

* Valores expressos em número de pacientes. 
Tabela III - Escala Subjetiva de Dor, em M0 e M1

\begin{tabular}{lcc}
\hline Escala de dor & M0* $^{*}$ & M1* $^{*}$ \\
\hline 0 & 8 & 12 \\
1 & 6 & 4 \\
2 & 2 & 0 \\
3 & 0 & 0 \\
4 & 0 & 0 \\
\hline
\end{tabular}

* Valores expressos em número de pacientes.

Tabela IV - Variações das Pressões Arteriais Sistólica e Diastólica M0 Versus M1

\begin{tabular}{lcccc}
\hline Variáveis & Média & Mediana & Desvio-Padrão & $\mathrm{p}$ \\
\hline VPS & $-29,4$ & -20 & 19,4 & 0.0001 \\
VPD & $-16,4$ & -19 & 11,09 & 0.0001 \\
\hline
\end{tabular}

VPS - variação da pressão arterial sistólica; VPD - variação da pressão arterial diastólica.

Tabela V - Variações da Duração do QRS

\begin{tabular}{lcccc}
\hline Variáveis & Média & Mínimo & Máximo & $\mathrm{p}$ \\
\hline Duração QRS & & & & \\
M0 & 104,7 & 78 & 140 & \\
M1 & 109,33 & 87 & 140 & \\
Variação QRS & 4,60 & -2 & 20 & 0,018 \\
\hline
\end{tabular}

Tabela VI - Variações do Intervalo Atrio-His*

\begin{tabular}{lcccc}
\hline Variáveis & Média & Mínimo & Máximo & $\mathrm{p}$ \\
\hline Intervalo AH & & & & \\
M0 & 95,92 & 70 & 115 & \\
M1 & 103,07 & 80 & 118 & \\
Variação AH & 7,13 & -19 & 12 & 0,006 \\
\hline
\end{tabular}

* Tempo de condução internodal atrioventricular, medido do eletrograma atrial ao eletrograma do feixe de His. Os valores normais em adultos variam de 35 a $150 \mathrm{~ms}$.

Quanto às variáveis de refratariedade cardíaca (período refratário do átrio direito, do ventrículo direito e do nódulo atrioventricular), houve aumento do período refratário do átrio direito $(p=0,001)$ e do período refratário do nódulo atrioventricular $(p=0,0001)$. Não foi observada diferença estatística significativa em relação ao período refratário do ventrículo direito.
Tabela VII - Variações do Tempo de Recuperação do Nódulo Sinoatrial ${ }^{*}$

\begin{tabular}{lcccc}
\hline Variáveis & Média & Mínimo & Máximo & $\mathrm{p}$ \\
\hline TRNS & & & & \\
M0 & 880,27 & 750,0 & $1.090,5$ & \\
M1 & 1070,7 & 900,0 & $1.490,7$ & \\
Variação TRNS & 195 & 528 & 3 & 0,0004 \\
\hline
\end{tabular}

* Tempo necessário para a recuperação da formação de um impulso sinusal após um superestímulo do marcapasso atrial. Valores normais em adultos variam de $<$ ou $=1.207 \mathrm{a}<\mathrm{ou}$ $=1.500$.

\section{DISCUSSÃO}

O remifentanil, assim como outros opióides, pode causar bradicardia e assistolia, antes ou durante a laringoscopia e intubação traqueal ${ }^{10-21}$.

Inicialmente esse quadro foi atribuído a mecanismo centralmente mediado; entretanto, sabe-se que a morfina apresenta um efeito direto no nódulo sinoatrial e na condução atrioventricular ${ }^{22,23}$. Já o sufentanil prolonga a duração do potencial de ação em fibras de Purkinje de cães ${ }^{24-26}$.

Da mesma forma, Reitan e col. atribuíram 10\% da bradicardia, causada pelo fentanil em cães, à sua ação periférica e não apenas vagotônica central ${ }^{27}$. Em ratos, Saeki e col. demonstram a ativação dos receptores opióides tipo mu no nódulo sinoatrial, o que comprovou um efeito cronotrópico negativo direto do mesmo ${ }^{28}$. No entanto, esses efeitos eletrofisiológicos diretos do fentanil não foram observados em humanos ${ }^{29}$.

Com o alfentanil e o sufentanil, Sharpe e col. também não demonstraram efeitos diretos na condução e na refratariedade cardíaca (estudo em humanos), ao contrário dos anestésicos inalatórios que aumentaram a refratariedade das vias de condução atrioventricular ${ }^{30,31}$.

Em relação ao remifentanil, alguns mecanismos foram propostos para explicar a causa da bradicardia e da hipotensão arterial, dentre eles a possível liberação de histamina após administração rápida do fármaco, um estímulo vagal centralmente mediado, a inibição central do tônus simpático, um efeito direto na condução e na refratariedade cardíaca ou uma causa multifatorial ${ }^{10}$.

Todavia, Sebel e col. provaram que as alterações hemodinâmicas observadas não se correlacionam com as alterações nas concentrações sangüíneas de histamina ${ }^{32}$. Atualmente, acredita-se que a diminuição da pressão arterial média causada pelo remifentanil seja devida à vasodilatação periférica e conseqüente diminuição da resistência vascular sistêmica, por mecanismo endotélio-dependente, relacionado com a liberação de prostaciclina e óxido nítrico ${ }^{33}$. Shinohara e col. demonstraram que em ratos com neuroeixo intacto (com a resposta do reflexo barorreceptor manti- 
da) a hipotensão arterial e a bradicardia, abruptas e de rápida resolução, devem-se à ação vagotônica central do remifentanil ${ }^{34}$. No entanto, hipotensão arterial e bradicardia, nesse caso de início gradual, também foram observadas nos animais desnervados (sem o reflexo barorreceptor ativo), o que afastou a responsabilidade do efeito vagotônico central como causa única da bradicardia. Como esses animais vagotomizados não apresentavam diminuição do tônus simpático, essa hipótese também foi afastada. Os autores propuseram como causa da bradicardia, uma ação cronotrópica negativa direta, por estimulação dos receptores mu no nódulo sinoatrial. Essa hipótese é corroborada pela administração prévia de naloxona (antagonista do receptor $\mathrm{mu}$ ) que abole a bradicardia nos animais baro-desnervados e vagotomizados ${ }^{34}$.

Ao estudar o efeito do remifentanil sobre a resposta hemodinâmica à intubação traqueal, Thompson e col. avaliaram também o efeito da administração prévia de um agente vagolítico (glicopirrolato $200 \mu \mathrm{g}$ ), com o objetivo de evitar bradicardia. Os autores concluíram que o glicopirrolato não aumentou a freqüência cardíaca basal, mas atenuou a bradicardia associada ao uso do remifentanil (incidência de $50 \%$ no grupo remifentanil $\times 10 \%$ no grupo remifentanil e glicopirrolato) ${ }^{11}$.

Sebel e col. observaram uma redução média de $20 \%$ da pressão arterial e da freqüência cardíaca, após doses de 2 a $30 \mu \mathrm{g} . \mathrm{kg}^{-1}$ por via venosa em bolus de remifentanil (administrados em 1 minuto), em pacientes tratados previamente com glicopirrolato ${ }^{32}$.

O presente estudo comprovou que o remifentanil exerce um efeito depressor na função do nódulo sinoatrial (refletido pelo prolongamento do tempo de recuperação do nódulo sinoatrial) e na condução intra-atrial (caracterizado pelo aumento do intervalo átrio-His), o que justifica per si o seu perfil bradicárdico ${ }^{35}$.

Entretanto, não foram observadas alterações na freqüência cardíaca basal, o que provavelmente deva-se às pequenas doses de remifentanil empregadas (as variações do intervalo AH e do TRNS, apresentaram diferença estatística significativa, mas ainda assim com números absolutos dentro da faixa da normalidade) ou ao número de pacientes estudados. A segunda hipótese é pouco provável, pois as variações do intervalo $\mathrm{A}-\mathrm{H}$ e do TRNS, foram muito diferentes no estudo estatístico ( $p=0,006$ e 0,0004, respectivamente).

O remifentanil também causou aumento do período refratário do átrio direito e do nódulo atrioventricular, o que sugere um potencial disritmogênico desse fármaco ${ }^{36}$. Porém, apesar das alterações observadas nos períodos refratários, os mesmos também se encontram dentro da faixa fisiológica.
Quando comparado com os outros opióides estudados anteriormente o remifentanil ${ }^{30,31}$, ao contrário do esperado, causou alterações das variáveis eletrofisiológicos, mesmo nas pequenas doses empregadas (doses para sedação consciente) e as alterações observadas no presente estudo podem ser atribuídas apenas ao uso do remifentanil, pois Sharpe e col. demonstram que o midazolam não altera as variáveis de condução e refratariedade cardíaca ${ }^{30}$.

Adicionalmente, pelos resultados desse estudo ficou comprovado que o remifentanil é um fármaco capaz de causar hipotensão arterial, como já havia sido demonstrado anteriormente ${ }^{10,11}$.

Em sedação e analgesia, estudos anteriores demonstraram que a associação de remifentanil (bolus de $0,5 \mu \mathrm{g} \cdot \mathrm{kg}^{-1} \mathrm{~min}^{-1}$, em 30 segundos, seguido de infusão venosa contínua, na dose de $0,05 \mu \mathrm{g} \cdot \mathrm{kg}^{-1} \mathrm{~min}^{-1}$ ) e midazolam (2 mg por via venosa) é eficaz, pois $66 \%$ dos pacientes não apresentaram dor ou desconforto durante os procedimentos, com um grau de sedação leve. Além disso, observou-se pouco efeito colateral ( $16 \%$ de náuseas e $2 \%$ de vômitos) ${ }^{37,38}$.

Portanto, não fossem os resultados desse estudo, o remifentanil seria o componente analgésico ideal nas sedações e analgesia para a realização de estudo eletrofisiológico intracardíaco percutâneo, ablação de disritmias por cateter de radiofreqüência, ou ambos. Mas o presente estudo indica que o remifentanil não deve ser usado nesses procedimentos, pois pode interferir na precisão das medidas eletrofisiológicas.

Desse estudo concluiu-se que o remifentanil apresentou um efeito eletrofisiológico na função do nódulo sinoatrial e na condução e refratariedade cardíaca (aumentou o tempo de recuperação do nódulo sinoatrial, a duração do intervalo QRS, o intervalo $\mathrm{AH}$ e os períodos refratários do átrio direito e do nódulo atrioventricular), o que em doses maiores que as estudas poderia causar bradicardia sinusal, assistolia e distúrbios de condução.

Portanto, o mesmo deve ser evitado em pacientes com doenças documentadas do nódulo sinoatrial, distúrbios de condução prévios e em usuários habituais de fármacos que afetem o cronotropismo e dromotropismo cardíaco, como, por exemplo, os bloqueadores do receptor beta-adrenérgico. Concluiu-se também que o remifentanil não é um bom fármaco a ser empregado como componente analgésico nas sedações e analgesia para realização de estudos eletrofisiológicos intracardíacos, pois pode interferir na interpretação dos seus resultados.

Neste estudo não foram demonstradas alteração na freqüência cardíaca basal, mas outros estudos devem ser realizados no futuro, com doses maiores do fármaco, para que se avalie sua segurança. 


\section{Glossário}

1) Intervalo átrio-His $(\mathrm{AH})$ : Estima o tempo de condução internodal atrioventricular, medido do eletrograma atrial ao eletrograma do feixe de His. Os valores normais em adultos variam de 35 a $150 \mathrm{~ms}$

2) Intervalo PA: É uma aproximação do tempo de condução intraatrial. É medido do começo da onda $\mathrm{P}$ ao começo da ativação atrial no eletrograma de His (início do potencial A). Os valores normais em adultos em ritmo sinusal variam de 9 a $150 \mathrm{~ms}$.

3) Intervalo His-Ventriculo (HV): É o tempo de condução entre o local onde se registra o potencial $\mathrm{H}$ (feixe de His proximal) e o início da ativação ventricular. Os valores normais em adultos variam de 10 a $55 \mathrm{~ms}$.

4) Tempo de recuperação do nódulo sinoatrial (TRNS): É o tempo requerido para a recuperação da formação de um impulso sinusal após um super-estímulo do marcapasso atrial. Valores normais em adultos variam de $<\mathrm{ou}=1.207 \mathrm{a}<\mathrm{ou}=1.500$.

5) Período refratário efetivo do átrio direito (PREAD): É o mais longo intervalo S1-S2 (estímulo artefato) que não resulta em despolarização atrial. Valores normais em adultos variam de 150 a $360 \mathrm{~ms}$. (dependentes da amplitude do ciclo empregado).

6) Período refratário efetivo do ventrículo direito (PREVD): É o mais longo intervalo S1-S2 que não consegue evocar uma resposta ventricular. Valores normais em adultos variam de 170 a $290 \mathrm{~ms}$

7) Período refratário efetivo do nódulo atrioventricular (PRENAV): É o mais longo intervalo A1-A2 medido no eletrograma do feixe de His que não se propaga para o feixe de His. Valores normais em adultos variam de 230 a $430 \mathrm{~ms}$.

\section{Prospective Study on the Repercus- sions of Low Doses of Remifentanil on Sinoatrial Function and in Cardiac Conduction and Refractory Period}

Simone Soares Leite, TSA, M.D.; Elizabeth Bessadas Penna Firme, TSA, M.D.; Márcia Santana Bevilaqua, TSA, M.D.; Leonel dos Santos Pereira, TSA, M.D.; Jacob Atié, M.D.

\section{INTRODUCTION}

Remifentanil is a selective mu receptor agonist, and its potency is similar to that of fentanyl. It has a methyl ester propanoic bond in its chemical structure that is hydrolyzed by non-specific plasma and tissue esterases (carboxy esterase), resulting in predictable and short action, which does not accumulate with repeated doses or when administered by continuous intravenous infusion (context-dependent halflife of 3 minutes). Its volume of distribution in central and to- tal compartments is also very low, with a beta half-life much lower than other opioids, besides a small $\mathrm{K}_{\mathrm{e}} \mathrm{O}$ half-live, responsible for its fast onset of action ${ }^{1-9}$.

This pharmacokinetic and pharmacodynamic profile is very attractive, especially in cases of short-duration procedures. However, its use is associated with hemodynamic instability, with hypotension and bradycardia ${ }^{10-14}$. Besides, there is a report in the literature of two cases of asystole after the use of remifentanil in anesthetic induction ${ }^{15,16}$.

Bradycardia has been reported, especially in patients taking beta-adrenergic receptor antagonists, patients with preexisting bradycardia, and in those who received a bolus dose of the drug (> $1 \mu \mathrm{g} \cdot \mathrm{kg}^{-1}$ in 30 seconds) ${ }^{12,13}$. However, one of the cases of asystole described affected a young patient without associated diseases who did not receive a bolus of the drug ${ }^{16}$.

The objective of this study was to evaluate, in human beings, whether the effects of remifentanil on cardiac conduction and refractoriness explains the bradycardia observed by other authors.

\section{METHODS}

After approval by the Ethics Committee on Research of the Hospital Universitário Clementino Fraga Filho, and after patients signed an informed consent, a prospective study was conducted with 16 adult patients, both genders, scheduled for elective, percutaneous, intracardiac electrophysiologic study, ablation of arrhythmias by radiofrequency catheter, or both.

Patients received sedation and analgesia in which the sedative chosen was midazolam.

Inclusion criteria included: ages ranging from 18 and 65 years, physical status ASA I to III, and weighing less than $100 \%$ above the ideal body weight.

Exclusion criteria included patients: taking benzodiazepines, antidepressants, illegal drugs, or central nervous system stimulants; beta-receptor antagonists or alfa ${ }_{2}$-receptor agonists; who were anesthetized or took opioids during the prior 48 hours; taking amiodarone (or when the drug was discontinued in the prior 45 days); with Myasthenia Gravis, closed angle glaucoma, or those who were not "on an empty stomach"; with psychiatric problems, severe pulmonary disease, congestive heart failure, unstable angina, or myocardial infarction in the prior six weeks; with severe cardiac blocks; patients with nephropathy or liver disease.

Antiarrhythmic drugs were discontinued for at least five halflives (except amiodarone) in all patients.

The intracardiac electrophysiological study was performed at the cardiac electrophysiology laboratory of the Hospital Clementino Fraga Filho.

Patients did not receive any pre-anesthetic medication.

After monitoring with a 12-lead electrocardiogram with the EPTRACER V0.73 system (ECG), pulse oximetry, non-inva- 
sive blood pressure (NIBP), capnograph (calibrated by the Cardiocap Ultima Datex Ohmeda monitor), and venipuncture in the left upper limb with an $18 \mathrm{G}$ catheter, $20 \mathrm{~mL} . \mathrm{kg}^{-1}$ of Ringer's lactate were administered over 10 to 15 minutes. Infusion was reduced to keep patent vein. A $Y$ connector (Polifix ${ }^{\circledR}-B$ Braun) was attached to the IV tubing and a 3-way tap (TAP-3 Embramed) was placed between the connector and the IV catheter.

Midazolam, $0.03 \mathrm{mg} . \mathrm{kg}^{-1}$ (ideal weight), was administered before the femoral punctures performed by the electrophysiologist.

At M0 (5 minutes after the administration of midazolam), the degree of sedation (Ramsay scale), severity of pain using a subjective scale from 0 to $4(0=$ no pain, $4=$ the worst pain ever felt), systolic (SBP) and diastolic (DBP) blood pressures, heart rate (HR) without electrical stimulation, respiratory rate $(\mathrm{RR})$, arterial oxygen saturation $\left(\mathrm{SpO}_{2}\right)$ by pulse oximetry with supplementary oxygen therapy $\left(5 \mathrm{~L} \cdot \mathrm{min}^{-1} \mathrm{O}_{2}\right.$ with a Hudson mask), expired $\mathrm{CO}_{2}$ by capnography $\left(\mathrm{P}_{\mathrm{ET}} \mathrm{CO} 2\right)$, cardiac conduction (duration of the QRS complex, and $\mathrm{AA}, \mathrm{AH}, \mathrm{HV}$ and PA intervals), sinoatrial node function (recovery of the sinoatrial node), and cardiac refractory period (refractory period of the right atrium, right ventricle, and atrioventricular node) were evaluated. Refer to the glossary for definitions.

Basal electrophysiological study was done with the simultaneous recording of the 12-lead electrocardiogram and intracavitary signs (EPTRACER V0.73) that were capture by three or four intravenous catheter-electrodes (quadripolar $6 \mathrm{~F}$ Biosense Webster $^{\circledR}$ ) placed in the upper region of the right atrium, adjacent to the His bundle, at the tip of the right ventricle, and in the coronary sinus. The basal duration of the QRS complex and basal intervals (AA, PR, PA, AH, HV, and QT intervals) were evaluated during sinus rhythm with a recording speed of $100 \mathrm{~mm} . \mathrm{seg}^{-1}$. ERPRA, ERPRV, and ERAVN were determined by decrescent extra-stimuli inserted over the continuous stimulation in cycles of 600,500 , and $430 \mathrm{~ms}$. The time of recovery of the sinoatrial node (TRSN) was determined by direct right atrium stimulation with cycles of 600,550 , 430 , and $380 \mathrm{~ms}$ for 1 minute in each level and a recording speed of $100 \mathrm{~mm} . \mathrm{seg}^{-1}$. Ten cycles were analyzed after the end of the stimulation, and the greatest post-stimulation interval was used to calculate TRSN. The configuration of the $P$ wave on the surface ECG and intraatrial electrocardiograms were used to determine whether the origin of the first beat after the end of the stimulation really originated in the sinoatrial node.

At $\mathrm{MO}$, patients who did not present $\mathrm{PA}, \mathrm{AH}$, and $\mathrm{HV}$ intervals within the normal limits standardized by the laboratory, were excluded from the study, as well as patients with WPW whose anterograde PRE of the accessory pathway was smaller than that of the AVN.

After the initial measurements, a bolus of remifentanil, $0.5 \mu \mathrm{g} \cdot \mathrm{kg}^{-1}$ (ideal body weight) was administered over 30 seconds, followed by a continuous intravenous infusion ( $0.05 \mu \mathrm{g} . \mathrm{kg}^{-1}$ of ideal body weight), in order to maintain a gra- de 2 to 4 sedation, by the Ramsay scale, and severity of pain from 0 to 1 . The dose of remifentanil could be adjusted in up to $\pm 50 \%$ to reach these goals. Patients who did not achieve these results received a continuous infusion of propofol, with or without a bolus of $0.5 \mathrm{mg} \cdot \mathrm{kg}^{-1}$ (ideal body weight), and were excluded from the study.

After evaluating hemodynamic and respiratory parameters (NIBP, HR, RR, $\mathrm{P}_{\mathrm{ET}} \mathrm{CO}_{2}$, and $\mathrm{SpO}_{2}$ ), the dose of remifentanil was decreased in up to $50 \%$ of the initial dose, when systolic BP had decreased by more than $15 \mathrm{mmHg}$ or it was below $80 \mathrm{mmHg}$, when the respiratory rate was below $8 \mathrm{bpm}$, $\mathrm{SpO}_{2}$ was less than $94 \%$ (with supplementary oxygen), or when the heart rate was below $40 \mathrm{bpm}$.

The same parameters were evaluated at M1 (after $20 \mathrm{mi}-$ nutes of the adjusted infusion of remifentanil).

After evaluating all parameters, continuation of the anesthesia was at the discretion of the anesthesiologist. The capability to induce sustained tachycardia was not evaluated. Paired Student $t$ test was used for comparative analysis of the parameters at M0 and M1; levels of $p<0.05$ were considered significant.

\section{RESULTS}

Table I shows the demographic data of patients in the study. The procedure was indicated for diagnosis of and ablation of AV nodal reentrant tachycardia (AVNRT), in seven cases, and Wolf-Parkinson-White syndrome, in the remaining nine patients, using a radiofrequency catheter.

The procedure lasted a mean of $78 \pm 25$ minutes (maximum of 128 and minimum of 40 minutes), and the mean total dose of remifentanil per procedure was $276 \pm 115 \mu \mathrm{g}$ (maximum of 520 and minimum of $140 \mu \mathrm{g}$ ), with a mean of $3.45 \pm 0.12 \mu \mathrm{g} \cdot \mathrm{min}^{-1}$.

The degree of sedation was satisfactory in every case, with a grade 2 to 3 by the Ramsay scale at M0 and 3 to 4 at M1 (Table II).

Table I - Demographic Data $(\mathrm{n}=16)$

\begin{tabular}{lc}
\hline Parameters & \\
\hline Age (years) & \\
Weight $(\mathrm{kg})^{*}$ & $66 \pm-6(25-26)$ \\
Height $(\mathrm{m})^{*}$ & $1.63 \pm-0.10(1.51-1.83)$ \\
Gender & \\
Male & $10(62.5 \%)$ \\
Female & $6(38.5 \%)$ \\
Physical status** & \\
ASA II & $10(62.5 \%)$ \\
ASA III & $6(38.5 \%)$ \\
\hline
\end{tabular}

* Values expressed as Mean \pm SD or Number.

${ }^{* *}$ Total number (\%). 
The severity of pain by the subjective scale varied from 0 to 2 at $\mathrm{M} 0$ and 0 to 1 at M1 (Table III). Those parameters were considered satisfactory and it was not necessary to administer propofol to any patient.

Of the hemodynamic parameters evaluated (NIBP, HR, $\mathrm{P}_{\mathrm{ET}} \mathrm{CO}_{2}$, and $\mathrm{SpO}_{2}$ ) at $\mathrm{MO}$ and $\mathrm{M} 1$, only systolic and diastolic blood pressures showed statistically significant differences (Table IV). There were no significant differences in HR between $\mathrm{M} 0$ and $\mathrm{M} 1$.

Among the parameters of cardiac conduction (duration of the QRS complex, AA, HA, HV, and PA intervals), we observed an increase in the duration of the QRS complex and $\mathrm{AH}$ interval (Tables $\mathrm{V}$ and $\mathrm{VI}$ ). The other parameters did not show statistically significant differences between M0 and M1.

There was also increase in TRNS (Table VII).

Regarding the parameters of cardiac refractoriness (refractory period of the right atrium, right ventricle, and atrioventri-

Table II - Ramsay Scale at M0 and M1

\begin{tabular}{lcc}
\hline Ramsay scale & M0 ${ }^{*}$ & M1 $^{*}$ \\
\hline 1 & 0 & 0 \\
2 & 6 & 0 \\
3 & 10 & 4 \\
4 & 0 & 12 \\
5 & 0 & 0 \\
6 & 0 & 0 \\
\hline
\end{tabular}

* Values expressed as number of patients.

Table III - Subjective Pain Scale at M0 and M1

\begin{tabular}{lcc}
\hline Pain scale & M0 * & M1 * \\
\hline 0 & 8 & 12 \\
1 & 6 & 4 \\
2 & 2 & 0 \\
3 & 0 & 0 \\
4 & 0 & 0 \\
\hline
\end{tabular}

* Values expressed as the number of patients.

Table IV - Variations in Systolic and Diastolic Blood Pressure in $\mathrm{M} 0$ versus $\mathrm{M} 1$

\begin{tabular}{lcccc}
\hline Parameters & Mean & Median & Standard Deviation & $\mathrm{p}$ \\
\hline VSP & -29.4 & -20 & 19.4 & 0.0001 \\
VDP & -16.4 & -19 & 11.09 & 0.0001 \\
\hline
\end{tabular}

VSP - variation in systolic blood pressure.

VDP - variation in diastolic blood pressure.
Table V - Variations in the duration of the QRS Complex

\begin{tabular}{lcccc}
\hline Parameters & Mean & Minimum & Maximum & $\mathrm{p}$ \\
\hline Duration of QRS & & & & \\
M0 & 104.7 & 78 & 140 & \\
M1 & 109.33 & 87 & 140 & \\
Variation of QRS & 4.60 & -2 & 20 & 0.018 \\
\hline
\end{tabular}

Table VI - Variations in the Atrium-His Interval*

\begin{tabular}{lcccc}
\hline Parameters & Mean & Minimum & Maximum & $p$ \\
\hline AH interval & & & & \\
M0 & 95.92 & 70 & 115 & \\
M1 & 103.07 & 80 & 118 & \\
Variation of AH & 7.13 & -19 & 12 & 0.006 \\
\hline
\end{tabular}

* Time of internodal atrioventricular conduction measured from the atrial electrogram to the electrogram of the His bundle. Normal values in adults range from 35 to $150 \mathrm{~ms}$.

Table VII - Variations in the Time of Recovery of the Sinoatrial Node*

\begin{tabular}{lcccc}
\hline Parameters & Mean & Minimum & Maximum & $\mathrm{p}$ \\
\hline TRSN & & & & \\
M0 & 880.27 & 750.0 & 1090.5 & \\
M1 & 1070.7 & 900.0 & 1490.7 & \\
Variation of TRSN & 195 & 528 & 3 & 0.0004 \\
\hline
\end{tabular}

* Time necessary for recuperation of the formation of a nodal impulse after a super-stimulus of the atrial pacemaker. Normal values in adults range from $<$ or $=1207$ to $<$ or $=1500$.

cular node), there was an increase in the refractory period of the right atrium ( $p=0.001)$ and atrioventricular node $(p=0.0001)$. There were no statistically significant differences in the refractory period of the right ventricle.

\section{DISCUSSION}

Similar to other opioids, remifentanil can cause bradycardia and asystole, before or during laryngoscopy and tracheal intubation ${ }^{10-21}$.

Initially, it was attributed to a central-mediated mechanism; however, Morphine is known to exert a direct effect on the sinoatrial node and atrioventricular conduction ${ }^{22,23}$. On the other hand, sufentanil prolongs the duration of the action potential in Purkinje fibers in dogs ${ }^{24-26}$.

Reitan et al. attributed $10 \%$ of the bradycardia caused by sufentanil in dogs to its peripheral effects, and not only to its 
central vagotonic effect ${ }^{27}$. Saeki et al. demonstrated, in mice, the activation of opioid mu receptors in the sinoatrial node and, therefore, proving a direct negative chronotropic action ${ }^{28}$. However, these direct electrophysiological effects of fentanyl were not observed in human beings ${ }^{29}$.

Sharpe et al. also did not demonstrate direct effects of alfentanil and sufentanil on cardiac conduction and refractoriness (in human beings), which is contrary to the effects of inhalational anesthetics that increase the refractoriness of atrioventricular conduction pathways ${ }^{30,31}$.

As for remifentanil, some mechanisms have been proposed to explain the bradycardia and hypotension caused by this drug; among them, we can include the possible release of histamine after fast administration of the drug, a centrallymediated vagal stimulus, central inhibition of the sympathetic tone, a direct effect on cardiac conduction and refractory period, or a multifactorial cause ${ }^{10}$.

However, Sebel et al. demonstrated that the hemodynamic changes observed are not related with changes in plasma levels of histamine ${ }^{32}$.

Currently, it is believed that the reduction in mean arterial blood pressure caused by remifentanil is secondary to the peripheral vasodilation, and consequent reduction in systemic vascular resistance, by an endothelium-dependent mechanism related to the release of prostacyclin and nitric oxide ${ }^{33}$.

Sinohara et al. showed that, in mice with intact neuron axis (preserved response to baroreceptor reflex), sudden and easily corrected hypotension and bradycardia are due to the central vagotonic action of remifentanil ${ }^{3,4}$. However, slowly developing hypotension and bradycardia were also observed on denervated animals (without active baroreceptor reflex), which showed that the vagotonic central effect is not the only cause of bradycardia. Since these animals did not show a reduction in sympathetic tone, this hypothesis was also ruled out. The authors proposed that bradycardia would be caused by a direct negative chronotropic effect secondary to direct stimulation of mu receptors in the sinoatrial node. This hypothesis is supported by the prior administration of Naloxone (a mu receptor antagonist), which abolishes bradycardia in baro-denervated and vagotomized animals ${ }^{3,4}$.

Thompson et al., studying the effects of remifentanil on hemodynamic response to tracheal intubation, also evaluated the effects of prior administration of a vagolytic agent (glycopyrrolate $200 \mu \mathrm{g}$ ), in an attempt to avoid the onset of bradycardia. The authors concluded that glycopyrrolate did not increase basal cardiac frequency, but it attenuated the bradycardia associated with the administration of remifentanil $(50 \%$ incidence in the remifentanil group $\times 10 \%$ in the remifentanil and glycopyrrolate group) ${ }^{11}$.

Sebel et al. observed a mean reduction of $20 \%$ in blood pressure and heart rate after the intravenous administration of remifentanil, in doses ranging from 2 to $30 \mu \mathrm{g} . \mathrm{kg}^{-1}$ (administered over 1 minute), in patients who received glycopyrrolate before remifentanil ${ }^{32}$.
The current study proved that remifentanil depresses the function of the sinoatrial node (reflected in an increase in the recovery period of the sinoatrial node) and in intra-atrial conduction (by an increase in the atrium-His interval) justifying, therefore, the bradycardia associated with the administration of this drug ${ }^{35}$.

However, we did not observe changes in basal heart rate, probably due to the small doses of remifentanil used in this study (the variations in $\mathrm{AH}$ interval and in TRSN were statistically significant but, in absolute numbers, within the normal range) or to the size of the study group. The second hypothesis is unlikely to be true, i.e., changes in $\mathrm{A}-\mathrm{H}$ interval and TRSN were very different statistically $(p=0.006$ and 0.0004 , respectively).

Remifentanil also caused an increase in the refractory period of the right atrium and atrioventricular node, suggesting an arrhythmogenic potential of this drug ${ }^{36}$. However, the changes observed in refractory periods are within normal limits.

Compared to other opioids studied ${ }^{30,31}$, even small doses of remifentanil (doses for awake sedation) caused changes in electrophysiological parameters, and the changes observed in the current study can be attributed to this drug since Sharpe et al. demonstrated that midazolam does not change cardiac conduction and refractoriness ${ }^{30}$.

Additionally, these results proved that remifentanil is capable of causing hypotension, as had been shown before ${ }^{10,11}$.

Regarding sedation and analgesia, other studies have demonstrated that the association of remifentanil (bolus of $0.5 \mu \mathrm{g} \cdot \mathrm{kg}^{-1} \cdot \mathrm{min}^{-1}$ over 30 seconds, followed by continuous intravenous infusion, $0.05 \mu \mathrm{g} \cdot \mathrm{min}^{-1}$ ) and midazolam (2 mg IV) is effective, since $66 \%$ of the patients did not experience pain or discomfort during the procedures, and provided light sedation. Besides, few side effects were observed $(16 \%$ of nausea and $2 \%$ of vomiting) 37,38 .

Therefore, remifentanil would be the ideal analgesic component in sedations and analgesia for percutaneous intracardiac electrophysiological studies, ablation of arrhythmias with radiofrequency catheter, or both, if it were not for the results of our study. These results suggest that remifentanil should not be used in those procedures, because it can interfere with the precision of electrophysiological measurements. From what has been exposed, we concluded that remifentanil had an electrophysiological effect in the function of the sinoatrial node and in cardiac conduction and refractoriness (it increased the recovery time of the sinoatrial node, the duration of the QRS complex, $\mathrm{AH}$ interval, and refractory periods of the right atrium and atrioventricular node), and, in higher doses that the ones used in this study, it could cause sinus bradycardia and conduction defects.

Therefore, its use should be avoided in patients with known disorders of the sinoatrial node, prior conduction disturbances, and in patients who take regularly drugs that affect cardiac chronotropism, such as beta-blockers.

We also concluded that remifentanil is not a good drug to be used as the analgesic component in sedation and analgesia 
for intracardiac electrophysiological studies because it can interfere with the interpretation of the results.

In this study, we did not observe changes in basal heart rate, but further studies should be performed in the future, with higher doses of the drug, to evaluate its safety.

\section{Glossary}

1) Atrium-His interval $(\mathrm{AH})$ - Estimates the time of internodal atrioventricular conduction, measuring from the atrial electrogram to the electrogram of the bundle of His. Normal values in adults vary from 35 to $150 \mathrm{~ms}$.

2) PA interval - An approximation of the intraatrial conduction time. It is measured from the beginning of the $P$ wave to the beginning of atrial activation in the His electrogram (beginning of the $A$ potential). Normal values in adults in sinus rhythm vary from 9 to $150 \mathrm{~ms}$.

3) His-ventricle interval (HV) - It is the conduction time between the place where the His potential is recorded (proximal bundle of $\mathrm{His}$ ) and the beginning of ventricular activation. Normal values in adults vary from 10 to $55 \mathrm{~ms}$.

4) Time of recovery of the sinoatrial node (TRSN) - Time required for recovery of sinus signal formation after a super-stimulation of the atrial pacemaker. Normal values in adults vary from $<$ or $=1207$ to $<$ or $=1500$.

5) Effective refractory period of the right atrium (ERPRA) - The longest S1-S2 interval (artifact stimulus) that does not result in atrial depolarization. Normal values in adults vary from 150 to $360 \mathrm{~ms}$ (dependent on the amplitude of the cycle used).

6) Effective refractory period of the right ventricle (ERPRV) - The longest S1-S2 interval that cannot evoke a ventricular response. Normal values for adults vary from 170 to $290 \mathrm{~ms}$.

7) Effective refractory period of the atrioventricular node (ERPAVN) - The longest A1-A2 interval measured on the His electrogram that does not propagate through the bundle of His. Normal values for adults vary from 230 to $430 \mathrm{~ms}$.

\section{REFERÊNCIAS - REFERENCES}

01. Glass PS, Gan TJ, Howell S - A review of the pharmacokinetics and pharmacodynamics of remifentanil. Anesth Analg, 1999; 89:S7-14.

02. Nora FS, Fortis EAF - Remifentanil: Por que precisamos de outro opióide? Rev Bras Anestesiol, 2001:51:146-159.

03. Shafer $\mathrm{SL}-\mathrm{New}$ intravenous anesthetic: Remifentanil. ASA Refresher Course, 1996;24:243-255.

04. Egan TD - Pharmacokinetics and pharmacodynamics of remifentanil: an update in the year 2000. Curr Opin Anaesthesiol, 2000;13:449-455.

05. Videira RLR, Cruz JRS - Remifentanil na prática clínica. Rev Bras Anestesiol, 2004;54:114-128.

06. Thompson JP, Rowboteram DJ - Remifentanil: an opióide for the $21^{\text {st }}$ century. Br J Anaesth, 1996;76:341-343.

07. Scott LJ, Perry CM - Spotlight on remifentanil for general anaesthesia. CNS Drugs 2005;19(12):1069-74.

08. Bürkle H, Dunbar S, Van Aken H - Remifentanil: A novel, shortacting, mu-opioid. Anesth Analg 1996;83:646-651.

09. Servin FS - Remifentanil: an update. Curr Opin Anaesthesiol, 2003;16:367-372.
10. Elliot P, O'Hare R, Bill KM et al. - Severe cardiovascular depression with remifentanil. Anesth Analg, 2000;91:58-61.

11. Thompson JP, May AP, Russell J et al. - Effect of remifentanil on the haemodynamic response to orotracheal intubation. $\mathrm{Br} \mathrm{J}$ Anaesth, 1998;80:467-469.

12. Reid JE, Mirakhur RK - Bradycardia after administration of remifentanil. Br J Anesth, 2000;84:422-423.

13. DeSouza G, Lewis MC, TerRiet MF et al. - Severe bradycardia after remifentanil. Anesthesiology, 1997;87:1019-1020.

14. Glass PSA - Pharmacology of remifentanil. Eur J Anaesthesiol, 1995;12(suppl.10):73-74.

15. Wang J, Winship S, Russell G - Induction of anaesthesia with sevoflurane and low-dose remifentanil: asystole following laryngoscopy. Br J Anestth, 1998; 81:994-995.

16. Altermatt FR, Muñoz HR - Asystole with propofol and remifentanil. Br J Anesth, 2000;84:696-697.

17. Starr NJ, Sethna DH, Estafanous FG - Bradycardia and asystole following the rapid administration of sufentanil with vecuronium. Anesthesiology, 1986;64:521-523.

18. Maryniak JK, Bishop VA - Sinus arrest after alfentanil. Br J Anaesth, 1987;59:390-391.

19. Sherman EP, Lebowitz PW, Street WC - Bradycardia following sufentanil-succinylcholine. Anesthesiology, 1987;66:106.

20. Rivard JC, Lebowitz PW - Bradycardia after alfentanilsuccinylcholine. Anesth Analg, 1988;67:907.

21. Egan TD, Brock-Utne JG - Asystole after anesthesia induction with fentanyl, propofol, and succinylcholine sequence. Anesth Analg, 1991;73:818-820.

22. Kennedy BL, West TC - Effect of morphine on electrically induced release of autonomic mediators in the rabbit sinoatrial node. J Pharmacol Exp Ther, 1967;157:149-158.

23. Tomichek RC, Rosow C, Philbin DM et al. - Diazepan-fentanyl interaction hemodynamic and hormonal effects in coronary artery surgery. Anesth Analg, 1983;62:881-884.

24. Blair JR, Pruett JK, Introna RPS et al. - Cardiac electrophysiologic effects of fentanyl and sufentanil in canine cardiac Purkinje fibers. Anesthesiology, 1989;71:565-570.

25. Puerto BA, Wong KC, Puerto AX et al. - Epinephrine-induced dysrhythmias: Comparison during anaesthesia with narcotics and with halogenated agents in dogs. Can Anaesth Soc J, 1979; 26:263-268.

26. Weber G, Stark G, Stark U - Direct cardiac electrophysiologic effects of sufentanil and vecuronium in isolated guinea-pig hearts. Acta Anaesthesiol Scand, 1995;39:1071-1074.

27. Reitan N, Stengert KB, Wymore ML et al. - Central vagal control of fentanyl-induced bradycardia during halothane anesthesia. Anesth Analg, 1978;57:31-36.

28. Saeki T, Nishimura M, Sato N - Electrophysiological demonstration and activation of mu-opioid receptors in rabbit sinoatrial node. J Cardiovasc Pharmacol, 1995;26:160-168.

29. Gómes-Arnau J, Márquez-Montes J, Avello F - Fentanyl and droperidol effects on refractoriness of acessory pathways in the Wolff-Parkinson-White syndrome. Anesthesiology, 1983;58:307313.

30. Sharpe MD., Dobkowski WB, Murkin JM et al. - Alfentanilmidazolam anaesthesia has no electrophysiological effects upon the normal conduction system or accessory pathways in patients with Wolff-Parkinson-White syndrome. Can J Anaesth, 1992;39:816-821.

31. Sharpe MD., Dobkowski WB, Murkin JM et al. - The electrophysiologic effects of volatile anesthetics and sulfentanil on the normal atrioventricular conduction system and accessory pathways in Wolff-Parkinson-White syndrome. Anesthesiology, 1994;80:63-70.

32. Sebel PS, Hoke JF, Westmoreland C et al. - Histamine concentrations and haemodynamic responses after remifentanil. Anesth Analg, 1995;80:990-993. 
33. Unlugenc $\mathrm{H}$, Itegin $\mathrm{M}$, Ocal I. - Remifentanil produces vasorelaxation in isolated rat thoracic aorta strips. Acta Anaesthesiol Scand, 2003;47:65-69.

34. Shinohara K, Aodo H, Uruh GK et al. - Suppressive effects of remifentanil on aerodynamics in baro-denervated rabbits. Can J Anaesth, 2000;47:361-366.

35. Josephson ME - Clinical Cardiac Eletrophysiology: Techniques and Interpretation, $2^{\text {nd }}$ Ed., Philadelphia, Lippincott Williams \& Wilkins,2002;6-166.

36. Atlee JL, Bosnjak ZJ - Mechanism for cardiac dysrhythimias during anesthesia. Anesthesiology, 1990;72:347-374.

37. Gold MI, Watkins W, David MD et al. - Remifentanil versus remifentanil/midazolam for ambulatory surgery during monitored anesthesia care. Anesthesiology, 1997;87:51-57.

38. Avramov MN, Smith IMB, White PF - Interactions between midazolam and remifentanil during monitored anesthesia care. Anesthesiology, 1996;85:1283-1289.

\section{RESUMEN}

Leite SS, Firme EBP; Bevilaqua MS, Pereira LS, Atié J - Estudio Prospectivo de las Repercusiones de Bajas Dosis de Remifentanil en la Función Sinoatrial en la Conducción y Refractariedad Cardiaca.

JUSTIFICATIVA Y OBJETIVOS: El remifentanil es un opioide con inicio y fin de acción rápidos, cuyo uso en procedimientos de corta duración se ha venido propagando en los últimos años. Entre los efectos colaterales descritos, hay relatos de bradicardia y asistolia. El objetivo de este estudio fue evaluar los efectos de este fármaco en la conducción y refractariedad cardíaca, en humanos.
MÉTODO: Estudio prospectivo de 16 pacientes, entre 18 y 65 años, de ambos sexos, ASA I a III, que se sometieron a estudio electrofisiológico intra cardiaco electivo. Se excluyeron pacientes con enfermedades del nódulo sino-atrial y los portadores de bloqueos cardíacos graves. En el laboratorio de electrofisiologia, los pacientes fueron inicialmente sedados con midazolam $\left(0,03 \mathrm{mg} \cdot \mathrm{kg}^{-1}\right)$, 5 minutos después (Mo) se evaluó el grado de sedación e intensidad de dolor, presiones arteriales sistólica y diastólica, frecuencias cardíaca y respiratoria y saturación de oxígeno. El electrofisiologista evaluó las variables de conducción cardíaca (duración del QRS, intervalos $A A, A H, H V$ y $P A)$, el tiempo de recuperación del nódulo sino-atrial y las variables de refractariedad cardíaca (período refractario del atrio derecho, período refractario del ventrículo derecho y período refractario del nódulo atrio ventricular). Después de las medidas iniciales el remifentanil fue introducido (bolo de $0,5 \mu \mathrm{g} \cdot \mathrm{kg}^{-1}+$ infusión de $0,05 \mu \mathrm{g} \cdot \mathrm{kg}^{-1} \cdot \mathrm{min}^{-1}$ ) y después de 20 minutos las mismas variables fueron evaluadas nuevamente (M1).

RESULTADOS: Se observó disminución de las presiones sistólica y diastólica $(p=0.0001)$ entre M0 y M1, sin diferencia estadística significativa de la frecuencia respiratoria o de la saturación de oxígeno. Hubo aumento del intervalo atrio-His $(p=0,006)$ y del tiempo de recuperación del nódulo sino-atrial $(p=0,0004)$, del período refractario del atrio derecho $(p=0,001)$ y del período refractario del nódulo atrio ventricular $(p=0,0001)$, pero no hubo disminución de la frecuencia cardíaca basal entre Mo e M1.

CONCLUSIONES: El remifentanil altero las variables electrofisiológicas cardíacas, lo que en dosis mayores que las estudiadas podría causar bradicardia sinusal, asistolia y disturbios de conducción. 